\title{
Two-dimensional telegraphic processes and their fractional generalizations
}

\author{
Jaume Masoliver ( ${ }^{*}$ \\ Department of Condensed Matter Physics and Institute of Complex Systems (UBICS), University of Barcelona, 08028 Catalonia, Spain \\ Katja Lindenberg (i) $^{\dagger}$ \\ Department of Chemistry and Biochemistry and BioCircuits Institute, University of California, San Diego 92093-0340, USA
}

(Received 23 October 2019; published 31 January 2020)

\begin{abstract}
We study the planar motion of telegraphic processes. We derive the two-dimensional telegrapher's equation for isotropic and uniform motions starting from a random walk model which is the two-dimensional version of the multistate random walk with a continuum number of states representing the spatial directions. We generalize the isotropic model and the telegrapher's equation to include planar fractional motions. Earlier, we worked with the one-dimensional version [Masoliver, Phys. Rev. E 93, 052107 (2016)] and derived the three-dimensional version [Masoliver, Phys. Rev. E 96, 022101 (2017)]. An important lesson is that we cannot obtain the twodimensional version from the three-dimensional or the one-dimensional one from the two-dimensional result. Each dimension must be approached starting from an appropriate random walk model for that dimension.
\end{abstract}

DOI: 10.1103/PhysRevE.101.012137

\section{INTRODUCTION}

Telegraphic processes generalize diffusion processes in two ways: (i) They allow for a finite velocity whereas diffusion processes are instantaneous at all points in space, implying an infinite speed. This is contrary to the principles of relativity and unsatisfactory from a conceptual point of view. (ii) Telegraphic processes are nearly deterministic (ballistic) in any direction at short times, whereas, at long times, they are diffusive. This characteristic is very useful, for instance, when modeling transport phenomena where thermalization due to random collisions takes a measurable time and the flux of ballistic particles may not be negligible, all of it resulting in anisotropic scattering along the forward direction [1]. For the same reason, telegraphic processes are useful in the description of early-time effects when ballistic motion may be important as is the case near interfaces.

As a first approximation, telegraphic processes can be described by the telegrapher's equation (TE), which can be written as

$$
\frac{\partial^{2} p}{\partial t^{2}}+\frac{1}{\tau} \frac{\partial p}{\partial t}=v^{2} \nabla^{2} p
$$

Here, $p=p\left(\mathbf{r}, t \mid \mathbf{r}_{0}\right)$ is the probability density function for the process to be at $\mathbf{r}$ at time $t$ having started at $\mathbf{r}_{0}$ at $t=0$, $\tau>0$ is a characteristic time, and $v>0$ is a characteristic speed. From a mathematical point of view, this is a hyperbolic equation which, as $\tau \rightarrow \infty$ with $v$ fixed, becomes the wave equation,

$$
\frac{\partial^{2} p}{\partial t^{2}}=v^{2} \nabla^{2} p
$$

\footnotetext{
*jaume.masoliver@ub.edu
}

†klindenberg@ucsd.edu whereas as $\tau \rightarrow 0$ and $v \rightarrow \infty$ such that $v^{2} \tau \rightarrow D$ is finite it reduces to the diffusion equation,

$$
\frac{\partial p}{\partial t}=D \nabla^{2} p
$$

Equation (1), thus, possesses wave and diffusion features, and it describes "diffusion with finite propagation velocity" but also "wave motion with damping" [2].

The TE first appeared in the nineteenth century in the works of Kelvin and of Heaviside related to the analysis of transmission of electromagnetic waves in telegraphic wires. In this context, the three-dimensional telegrapher's equation can be derived directly by combining Maxwell's equations for homogeneous media [1,2]. It can also be phenomenologically derived from thermodynamics by using Cattaneo's equation, a nonlocal generalization of Fick's law accounting for noninstantaneous diffusions [3-5], and from random walk theory where the one-dimensional TE is the master equation of the persistent random walk [6-8] (see also Ref. [9] for a fractional version of the one-dimensional TE and Ref. [10] for a recent three-dimensional generalization).

From a mesoscopic point of view (somewhere between the microscopic view of random walk models and the macroscopic approach of thermodynamics) telegraphic processes are closely related to Brownian motion. As was studied some years ago in Refs. [11-13], the telegrapher's equation, like the diffusion equation, can also be derived from the Chapman-Kolmogorov equation, which is the master equation for Markovian processes.

In the context of transport theory, the three-dimensional TE is the so-called $P_{1}$ approximation to the full transport equation for which the basic assumption is that the change in the direction of motion due to a single scattering event is small [1,14-16]. In a more recent approach [17], a threedimensional TE model is obtained by a modification of the 
continuity equation for the probability current. The model is, however, limited to a discrete number of transport directions, which restricts possible applications. Other approaches suppose phenomenological generalizations where a threedimensional TE is postulated for uniform isotropic media by assuming the same form as the one-dimensional TE but with numerical corrections in the coefficients which guarantee correct ballistic $(t \rightarrow 0)$ and diffusive $(t \rightarrow \infty)$ behaviors in three dimensions [18-20]. The more fundamental and less phenomenological way of describing telegraphic processes is, however, based on random walk models since they try to reproduce the microscopic mechanism of transport.

Random walk models for describing telegraphic processes are modifications of the ordinary random walk because the latter for long times and long distances (i.e., the so-called "fluid limit" [21]) lead to the diffusion equation but not to the telegrapher's equation $[1,2,22]$. However, and contrary to one dimension where the TE is readily obtained from the persistent random walk on the line [1,6,7], in higher dimensions obtaining the TE from microscopic models encounters serious difficulties. The main reason lies in the difficulty of generalizing persistence in dimensions greater than one [23-29].

We have recently solved this problem by obtaining the three-dimensional TE from a random walk model [10]. The model-a generalization of the persistent random walk in three dimensions - consists of a continuous version of a threedimensional random walk with a continuum of states [10]. However, the TE obtained is only valid for three dimensions and not for any other number of dimensions greater than one. Since dimensions other than three, especially bidimensional problems, are also important in many practical applications, we will herein obtain the two-dimensional TE from a planar random walk model.

There are several physical situations in which twodimensional diffusion processes and, hence, two-dimensional telegraphic processes are of great interest. In this way, the transport of particles on surfaces has a great number of physical applications in a large variety of problems including, for instance, semiconductor electronics [30], interfacial transport [31-35], chemical physics [34-38], and biophysical motions on surfaces $[39,40]$ among many others.

In our recent approaches to persistent random walks giving rise to one-dimensional or three-dimensional telegrapher's equations $[9,10]$, one of us has also generalized the procedures to include fractional versions of the TE that provide one mechanism for anomalous transport and related phenomena. In Ref. [10], we have proved that the fractional TE in threedimensional uniform isotropic media reads

$$
\frac{\partial^{2 \alpha} p}{\partial t^{2 \alpha}}+\frac{1}{\tau} \frac{\partial^{\alpha} p}{\partial t^{\alpha}}=v^{2} \nabla^{2 \gamma} p
$$

Here, $\partial^{\alpha} p / \partial t^{\alpha}$ is the Caputo derivative, and $\nabla^{2 \gamma} p$ is the RieszFeller Laplacian $(0<\alpha \leqslant 1,0<\gamma \leqslant 1)$ [10]. Our objective here is to obtain the fractional telegrapher's equation (FTE) in two dimensions.

In one dimension, the FTE is mostly based on a fractional generalization of the persistent random walk [9]. We have also showed that straight higher-dimensional generalizations of the one-dimensional persistent random walk leads to inconsistent and paradoxical results [9], and we have had to use the continuous and isotropic three-dimensional random walk model to obtain a three-dimensional FTE [10]. Another objective of this paper is, therefore, to obtain the two-dimensional FTE starting with the two-dimensional isotropic random walk which, as mentioned above, is not included in the three-dimensional walk as a special case. ${ }^{1}$

The paper is organized as follows. In Sec. II, we present the general model in two dimensions, and in Sec. III, we solve it in isotropic and uniform media. The fluid limit approximation and the two-dimensional telegrapher's equations are derived in Secs. IV and V. In Sec. VI, we generalize the isotropic random walk in order to include a fractional version of it and derive the FTE in two dimensions. Concluding remarks are in Sec. VII.

\section{CONTINUOUS MULTISTATE RANDOM WALK IN TWO DIMENSIONS}

We present a microscopic model for the transport of particles in planar continuous media. The model is a twodimensional modification of the three-dimensional random walk derived in Ref. [10], and we basically follow that derivation. Imagine a two-dimensional walker moving along a straight line with a direction determined by an angle $\varphi$. At random instants of time, the walker shifts direction and, hence, the duration of the motion along a given $\varphi$ (the motion along a fixed angle $\varphi$ is usually called a sojourn) is a random variable determined by a probability density function (PDF) which we denote by $\psi(t \mid \varphi)$. The cumulative distribution,

$$
\Psi(t \mid \varphi)=\int_{t}^{\infty} \psi\left(t^{\prime} \mid \varphi\right) d t^{\prime}
$$

gives the probability that the duration of a given sojourn is greater than $t$.

In order to characterize the random walk, we also need the joint PDF for the displacement in a given sojourn along direction $\varphi$ to be equal to $\mathbf{r}=(x, y)$ and the sojourn duration to equal $t$. We denote this density as $h(\mathbf{r}, t \mid \varphi)$ and as $H(\mathbf{r}, t \mid \varphi)$ the probability density for the displacement to be $\mathbf{r}$ when the duration is greater than $t$. Observe that the duration PDF $\psi(t|| \varphi)$ is the marginal density of $h(\mathbf{r}, t \mid \varphi)$,

$$
\int_{\mathbb{R}^{2}} h(\mathbf{r}, t \mid \varphi) d^{2} \mathbf{r}=\psi(t \mid \varphi)
$$

\footnotetext{
${ }^{1}$ In one dimension, one attempt to give physical grounds for the time-fractional TE was the work of Refs. [41,42] (see also Ref. [43]) who, starting from Cattaneo's equation, proposed three different candidates for the one-dimensional time-fractional TE. One of them, having the standard form given in Eq. (4) for one dimension, is derived from the continuous time random walk formalism applied to the probability flux and followed by the particular choice of a Gaussian distribution for jump lengths [41]. In Ref. [9], we undertook a more general approach based on the persistent random walk without assuming any special form of the jump density and which results in a space-time fractional TE agreeing with the standard form.
} 
and $\Psi(t \mid \varphi)$ is the marginal probability stemming from the density $H(\mathbf{r}, t \mid \varphi)$,

$$
\int_{\mathbb{R}^{2}} H(\mathbf{r}, t \mid \varphi) d^{2} \mathbf{r}=\Psi(t \mid \varphi) .
$$

At the end of a given sojourn, the walker moving along direction $\varphi^{\prime}$ switches to direction $\varphi$ within $d \varphi$. We indicate, by $b\left(\varphi \mid \varphi^{\prime}\right)$, the probability density of this transition,

$$
b\left(\varphi \mid \varphi^{\prime}\right) d \varphi=\operatorname{Prob}\left\{\varphi^{\prime} \rightarrow \varphi+d \varphi\right\} .
$$

Let us denote by $p(\mathbf{r}, t, \varphi)$ the joint PDF for the walker to be at $\mathbf{r}$ at time $t$ and moving in direction $\varphi$. Our final objective is, however, to know the density $p(\mathbf{r}, t)$ for the random walker to be at $\mathbf{r}$ at time $t$ regardless of the direction. The latter is the marginal density of the former,

$$
p(\mathbf{r}, t)=\int_{0}^{2 \pi} p(\mathbf{r}, t, \varphi) d \varphi .
$$

In order to evaluate $p(\mathbf{r}, t, \varphi)$, we define the auxiliary density $\rho(\mathbf{r}, t, \varphi)$ as

$$
\begin{aligned}
\rho(\mathbf{r}, t, \varphi) d^{2} \mathbf{r} d t= & \operatorname{Prob}\{\text { a sojourn in direction } \varphi \text { ends in } \\
& \text { the region }(\mathbf{r}, \mathbf{r}+d \mathbf{r}) \text { at }(t, t+d t)\} .
\end{aligned}
$$

This joint density describes the state of the process at the scattering points where the direction of the walker changes. Thus, if a scattering event happens at time $t$, it must either be the first one (assuming the initial one to have occurred at $t=0)$ or else be an earlier change in direction $\varphi^{\prime} \rightarrow \varphi$ [ruled by $b\left(\varphi \mid \varphi^{\prime}\right)$ ] happened at any earlier time $t^{\prime}<t$ with the random walker at some position $\mathbf{r}^{\prime}$. It is not difficult to convince oneself that this renewal argument leads to the following integral equation for the auxiliary density:

$$
\begin{aligned}
\rho(\mathbf{r}, t, \varphi)= & b(\varphi) h(\mathbf{r}, t \mid \varphi)+\int_{0}^{2 \pi} b\left(\varphi \mid \varphi^{\prime}\right) d \varphi^{\prime} \\
& \times \int_{0}^{t} d t^{\prime} \int_{\mathbb{R}^{2}} h\left(\mathbf{r}-\mathbf{r}^{\prime}, t-t^{\prime} \mid \varphi\right) \rho\left(\mathbf{r}^{\prime}, t^{\prime}, \varphi^{\prime}\right) d^{2} \mathbf{r}^{\prime},
\end{aligned}
$$

where $b(\varphi)$ is the probability that the process starts moving in direction $\varphi$.

In terms of the auxiliary density $\rho(\mathbf{r}, t, \varphi)$, the PDF $p(\mathbf{r}, t, \varphi)$ for the walker to be at $\mathbf{r}$ at time $t$ whereas moving in direction $\varphi$ is

$$
\begin{aligned}
p(\mathbf{r}, t, \varphi)= & b(\varphi) H(\mathbf{r}, t \mid \varphi)+\int_{0}^{2 \pi} b\left(\varphi \mid \varphi^{\prime}\right) d \varphi^{\prime} \\
& \times \int_{0}^{t} d t^{\prime} \int_{\mathbb{R}^{2}} H\left(\mathbf{r}-\mathbf{r}^{\prime}, t-t^{\prime} \mid \varphi\right) \rho\left(\mathbf{r}^{\prime}, t^{\prime}, \varphi^{\prime}\right) d^{2} \mathbf{r}^{\prime}
\end{aligned}
$$

The reasoning behind this equation is similar to the one given for obtaining Eq. (9). The displacement of the walker is either within the first sojourn, this given by $b H$ on the right-hand side, or else an earlier change of direction occurred at time $t^{\prime}<t$ while the walker was at position $\mathbf{r}^{\prime}$, and the time interval to the next scattering exceeded $t-t^{\prime}$.

We, thus, see that, in the most general case, the solution to the problem, that is to say, obtaining the $\operatorname{PDF} p(\mathbf{r}, t)$ [cf. Eq. (8)] is given by first solving the integral equation (9) for the auxiliary function $\rho$ and afterwards substituting this solution into Eq. (10) and the result into Eq. (8). In the most general case, for arbitrary forms of $b\left(\varphi \mid \varphi^{\prime}\right), h(\mathbf{r}, t \mid \varphi)$, and $H(\mathbf{r}, t \mid \varphi)$, obtaining analytical expressions is out of reach, and one has to resort to numerical work.

\section{Independent scattering}

In order to proceed further analytically, we will assume as in the three-dimensional case [10], that, after each scattering event, the direction is randomized independently of the previous direction of the walker. This leads to the following form of the scattering kernel:

$$
b\left(\varphi \mid \varphi^{\prime}\right)=b(\varphi)
$$

which is independent of the incoming direction $\varphi^{\prime}$. The scattering process is, thus, an independent random process in the change of direction. In this case, Eqs. (9) and (10) reduce to

$$
\begin{aligned}
\rho(\mathbf{r}, t, \varphi)= & b(\varphi)\left[h(\mathbf{r}, t \mid \varphi)+\int_{0}^{t} d t^{\prime} \int_{\mathbb{R}^{2}} h\left(\mathbf{r}-\mathbf{r}^{\prime}, t-t^{\prime} \mid \varphi\right)\right. \\
& \left.\times d^{2} \mathbf{r}^{\prime} \int_{0}^{2 \pi} \rho\left(\mathbf{r}^{\prime}, t^{\prime}, \varphi^{\prime}\right) d \varphi^{\prime}\right],
\end{aligned}
$$

and

$$
\begin{aligned}
p(\mathbf{r}, t, \varphi)= & b(\varphi)\left[H(\mathbf{r}, t \mid \varphi)+\int_{0}^{t} d t^{\prime} \int_{\mathbb{R}^{2}} H\left(\mathbf{r}-\mathbf{r}^{\prime}, t-t^{\prime} \mid \varphi\right)\right. \\
& \left.\times d^{2} \mathbf{r}^{\prime} \int_{0}^{2 \pi} \rho\left(\mathbf{r}^{\prime}, t^{\prime}, \varphi^{\prime}\right) d \varphi^{\prime}\right] .
\end{aligned}
$$

Integrating Eqs. (12) and (13) with respect to all possible directions, recalling Eq. (8) and defining

$$
\begin{gathered}
\rho(\mathbf{r}, t)=\int_{0}^{2 \pi} \rho(\mathbf{r}, t, \varphi) d \varphi \\
h(\mathbf{r}, t)=\int_{0}^{2 \pi} b(\varphi) h(\mathbf{r}, t \mid \varphi) d \varphi
\end{gathered}
$$

[with a similar definition for $H(\mathbf{r}, t)$ ], we obtain a simpler integral equation for $\rho(\mathbf{r}, t)$,

$$
\rho(\mathbf{r}, t)=h(\mathbf{r}, t)+\int_{0}^{t} d t^{\prime} \int_{\mathbb{R}^{2}} h\left(\mathbf{r}-\mathbf{r}^{\prime}, t-t^{\prime}\right) \rho\left(\mathbf{r}^{\prime}, t^{\prime}\right) d^{2} \mathbf{r}^{\prime},
$$

and the PDF $p(\mathbf{r}, t)$ will be given by

$$
p(\mathbf{r}, t)=H(\mathbf{r}, t)+\int_{0}^{t} d t^{\prime} \int_{\mathbb{R}^{2}} H\left(\mathbf{r}-\mathbf{r}^{\prime}, t-t^{\prime}\right) \rho\left(\mathbf{r}^{\prime}, t^{\prime}\right) d^{2} \mathbf{r}^{\prime} .
$$

The problem can now be solved in Fourier-Laplace space. Thus, defining the joint Fourier and Laplace transform,

$$
\hat{\tilde{\rho}}(\boldsymbol{\omega}, s, \varphi)=\int_{0}^{\infty} e^{-s t} d t \int_{\mathbb{R}^{2}} e^{i \omega \cdot \mathbf{r}} \rho(\mathbf{r}, t, \varphi) d^{2} \mathbf{r},
$$

the integral equation (16) turns into a simple algebraic equation for $\hat{\rho}$ whose solution can be readily obtained and reads

$$
\hat{\tilde{\rho}}(\omega, s)=\frac{\hat{\tilde{h}}(\boldsymbol{\omega}, s)}{1-\hat{\tilde{h}}(\boldsymbol{\omega}, s)} .
$$


On the other hand, by transforming Eq. (17), we get

$$
\hat{\tilde{p}}(\boldsymbol{\omega}, s)=\hat{\tilde{H}}(\boldsymbol{\omega}, s)[1+\hat{\tilde{\rho}}(\boldsymbol{\omega}, s)],
$$

which, after substituting for (18), yields

$$
\hat{\tilde{p}}(\omega, s)=\frac{\hat{\tilde{H}}(\omega, s)}{1-\hat{\tilde{h}}(\omega, s)} .
$$

The form of Eq. (19) is equal to that of the equivalent PDF of the three-dimensional walk presented in Ref. [10] and it is a generalization of the Montroll-Weiss equation [44,45] for higher-dimensional continuous time random walks with independent directions.

\section{THE ISOTROPIC AND UNIFORM RANDOM WALK}

Equation (19) constitutes the formal solution to the problem for independent scattering in Fourier-Laplace space. It is valid for any form of the conditional densities $h(\mathbf{r}, t \mid \varphi)$ and $H(\mathbf{r}, t \mid \varphi)$. In other words, Eq. (19) applies to any kind of motion inside a given sojourn and to any distribution of sojourn times. In order to proceed further, and solve the problem in an explicit way by obtaining the explicit expression for $p(\mathbf{r}, t)$ in real time and space, we next assume that the walker moves in an isotropic medium so that the pausing time density and its cumulative probability are independent of the direction,

$$
\psi(t \mid \varphi)=\psi(t), \quad \Psi(t \mid \varphi)=\Psi(t) .
$$

We further assume that, inside any sojourn, the motion is uniform with a constant speed $c$ so that after each sojourn the velocity of the walker takes a different direction but with the same modulus and, hence, the kinetic energy is conserved. Despite its simplicity, the model describes the planar motion of noninteracting particles - such as, for instance, photonsundergoing elastic dispersion with randomly distributed fixed centers.

The uniform motion leads us to assume that the conditional densities for the displacement in a given sojourn are given by

$$
h(\mathbf{r}, t \mid \varphi)=\delta(\mathbf{r}-c \mathbf{u} t) \psi(t),
$$

and

$$
H(\mathbf{r}, t \mid \varphi)=\delta(\mathbf{r}-c \mathbf{u} t) \Psi(t),
$$

where $\mathbf{u}=(\cos \varphi, \sin \varphi)$ is the unit vector pointing in direction $\varphi$. The Fourier transforms of these densities read

$$
\tilde{h}(\boldsymbol{\omega}, t \mid \varphi)=e^{i(\boldsymbol{\omega} \cdot \mathbf{u}) c t} \psi(t),
$$

and

$$
\tilde{H}(\omega, t \mid \varphi)=e^{i(\omega \cdot \mathbf{u}) c t} \Psi(t) .
$$

In addition to the assumption that after each scattering event the new direction is randomized independently of the previous direction [cf. Eq. (11)], we also suppose complete isotropy so that all outgoing directions are equally likely. Therefore,

$$
b\left(\varphi \mid \varphi^{\prime}\right)=b(\varphi)=\frac{1}{2 \pi} .
$$

Let us recall that the average,

$$
\tilde{h}(\omega, t)=\int_{0}^{2 \pi} b(\varphi) \tilde{h}(\omega, t \mid \varphi) d \varphi
$$

represents the characteristic function of displacement and duration inside any sojourn independent of the direction of the walker [see Eq. (15)]. For the isotropic case and for uniform motion [cf. Eqs. (22) and (24)], we, thus, obtain

$$
\begin{aligned}
\tilde{h}(\boldsymbol{\omega}, t) & =\frac{\psi(t)}{2 \pi} \int_{0}^{2 \pi} e^{i c t \boldsymbol{\omega} \cdot \mathbf{u}} d \varphi \\
& =\frac{\psi(t)}{2 \pi} \int_{0}^{2 \pi} e^{i c t|\omega| \cos \varphi} d \varphi \\
& =\frac{\psi(t)}{\pi} \int_{0}^{\pi} \cos (c t|\omega| \cos \varphi) d \varphi .
\end{aligned}
$$

From the integral representation of the Bessel function $J_{0}(z)$ [46],

$$
J_{0}(c t|\omega|)=\frac{1}{\pi} \int_{0}^{\pi} \cos (c t|\omega| \cos \varphi) d \varphi,
$$

we get

$$
\tilde{h}(\omega, t)=\psi(t) J_{0}(c t|\omega|),
$$

and analogously

$$
\tilde{H}(\omega, t)=\Psi(t) J_{0}(c t|\omega|) .
$$

In order to proceed further, we should specify the form of the sojourn time density $\psi(t)$. One of the most natural and universal assumptions consists of taking the random instants of time at which the scattering process occurs to be Poissonian, which implies that the time intervals for the duration of any sojourn are exponentially distributed

$$
\psi(t)=\lambda e^{-\lambda t} \quad \text { and } \quad \Psi(t)=e^{-\lambda t},
$$

where $\lambda^{-1}$ is the mean sojourn time. We then have

$$
\tilde{h}(\boldsymbol{\omega}, t)=\lambda e^{-\lambda t} J_{0}(c t|\omega|), \quad \tilde{H}(\boldsymbol{\omega}, t)=\frac{1}{\lambda} \tilde{h}(\boldsymbol{\omega}, t) .
$$

Let us now take the Laplace transforms of these expressions. Recalling that

$$
\mathcal{L}\left\{J_{0}(c t|\omega|)\right\}=\frac{1}{\sqrt{s^{2}+c^{2} t^{2}|\omega|^{2}}},
$$

and the standard property [47],

$$
\mathcal{L}\left\{e^{-\lambda t} f(t)\right\}=\hat{f}(\lambda+s),
$$

we get

$$
\hat{\tilde{h}}(\omega, s)=\frac{\lambda}{\sqrt{(\lambda+s)^{2}+c^{2}|\omega|^{2}}}
$$

and

$$
\hat{\tilde{H}}(\omega, s)=\frac{1}{\sqrt{(\lambda+s)^{2}+c^{2}|\omega|^{2}}} .
$$

Substituting these expressions into Eq. (19), we finally obtain

$$
\hat{\tilde{p}}(\omega, s)=\frac{1}{\sqrt{(\lambda+s)^{2}+c^{2}|\omega|^{2}}-\lambda},
$$


which is the solution of the homogeneous and isotropic random walk on the plane and it is the starting point for deriving the two-dimensional telegrapher's equation as we will see in the following sections.

\section{FLUID LIMIT APPROXIMATION}

The homogeneous and isotropic random walk described above is a microscopic model of two-dimensional transport in which particles move along straight lines with constant speed until they elastically collide with fixed centers of force randomly distributed on a two-dimensional region. In order to obtain a telegrapher's equation, we need to zoom out of this microscopic picture by performing the "fluid limit" and rewrite the model for long times and long distances $[21,48]$. Because of Tauberian theorems [49,50], long times and long distances, $t \rightarrow \infty$ and $|\mathbf{r}| \rightarrow \infty$, correspond to small Laplace and Fourier variables $s \rightarrow 0$ and $|\boldsymbol{\omega}| \rightarrow 0$.

Let us note that we can get an approximate expression for the transformed PDF $\hat{\tilde{p}}(|\omega|, s)$ either through Eq. (31) or else through the approximate expressions of $\hat{\tilde{h}}$ and $\hat{\tilde{H}}$ given by Eqs. (29) and (30) for small $|\omega|$ and $s$ and their subsequent substitution into Eq. (19). We will follow the latter method since it will be useful for the fractional generalization of the random walk.

We start from Eq. (29) and rewrite it as

$$
\hat{\tilde{h}}(\omega, s)=\left(\frac{\lambda}{\lambda+s}\right)\left[1+\left(\frac{c|\omega|}{\lambda+s}\right)^{2}\right]^{-1 / 2},
$$

which, when $|\omega| \rightarrow 0$, can be written as

$$
\hat{\tilde{h}}(\omega, s)=\frac{\lambda}{\lambda+s}\left[1-\frac{1}{2}\left(\frac{c|\omega|}{\lambda+s}\right)^{2}+O\left(|\omega|^{4}\right)\right] .
$$

Thus,

$$
\begin{aligned}
1-\hat{\tilde{h}}(\omega, s) & =1-\frac{\lambda}{\lambda+s}\left[1-\frac{1}{2}\left(\frac{c|\omega|}{\lambda+s}\right)^{2}+O\left(|\omega|^{4}\right)\right] \\
& =\frac{1}{\lambda+s}\left[s+\frac{\lambda}{2}\left(\frac{c|\omega|}{\lambda+s}\right)^{2}+O\left(|\omega|^{4}\right)\right] \\
& =\frac{1}{(\lambda+s)^{3}}\left[s(\lambda+s)^{2}+\frac{\lambda}{2} c^{2}|\omega|^{2}+O\left(|\omega|^{4}\right)\right],
\end{aligned}
$$

and as $s \rightarrow 0$, we may write

$$
\begin{aligned}
1 & -\hat{\tilde{h}}(\omega, s) \\
& =\frac{1}{(\lambda+s)^{3}}\left[s\left(\lambda^{2}+2 \lambda s\right)+\frac{\lambda}{2} c^{2}|\omega|^{2}+O\left(s^{3},|\omega|^{4}\right)\right] .
\end{aligned}
$$

Substituting Eqs. (32) and (33) into Eq. (19) and recalling that $\hat{\tilde{H}}=\hat{\tilde{h}} / \lambda$, we obtain

$$
\hat{\tilde{p}}(\omega, s)=\frac{(\lambda+s)^{2}-(c|\omega|)^{2} / 2+O\left(|\omega|^{4}\right)}{s\left(\lambda^{2}+2 \lambda s\right)+\lambda(c|\omega|)^{2} / 2+O\left(s^{3},|\omega|^{4}\right)} .
$$

In order to ensure the stability of Eq. (34) under FourierLaplace inversion [and, hence, for the existence of a valid approximation for $p(\mathbf{r}, t)]$, it is necessary that the powers of $s$ and $|\omega|$ which appear in the numerator of Eq. (34) be less than the corresponding powers of the denominator [49]. We, therefore, write

$$
\hat{\tilde{p}}(\omega, s)=\frac{\lambda^{2}+2 \lambda s+O\left(s^{2},|\omega|^{2}\right)}{s\left(\lambda^{2}+2 \lambda s\right)+\lambda(c|\omega|)^{2} / 2+O\left(s^{3},|\omega|^{4}\right)},
$$

that is,

$$
\hat{\tilde{p}}(\omega, s)=\frac{\lambda / 2+s+O\left(s^{2},|\omega|^{2}\right)}{s(\lambda / 2+s)+c^{2}|\omega|^{2} / 4+O\left(s^{3},|\omega|^{4}\right)},
$$

and take as a fluid limit approximation of the PDF the expression,

$$
\hat{\tilde{p}}(\omega, s)=\frac{s+\lambda / 2}{s(s+\lambda / 2)+c^{2}|\omega|^{2} / 4} .
$$

\section{THE TWO-DIMENSIONAL TELEGRAPHER'S EQUATION}

Equation (35) is the starting point for deriving the twodimensional TE. As in the three-dimensional case [10], we will obtain an associated partial differential equation for $p(\mathbf{r}, t)$ whose solution, in Fourier-Laplace space and with appropriate initial conditions, is precisely given by Eq. (35). To this end, we multiply both sides of Eq. (35) by the denominator and write

$$
s^{2} \hat{\tilde{p}}(\omega, s)-s+\frac{\lambda}{2}[s \hat{\tilde{p}}(\omega, s)-1]=-\frac{c^{2}}{4}|\omega|^{2} \hat{\tilde{p}}(\omega, s) .
$$

Recalling that

$$
\mathcal{F}^{-1}\left\{|\omega|^{2} \hat{\tilde{p}}(\boldsymbol{\omega}, s)\right\}=-\nabla^{2} \hat{p}(\mathbf{r}, s), \quad \mathcal{F}^{-1}\{1\}=\delta(\mathbf{r}),
$$

the Fourier inversion yields

$$
s^{2} \hat{p}(\mathbf{r}, s)-s \delta(\mathbf{r})+\frac{\lambda}{2}[s \hat{p}(\mathbf{r}, s)-\delta(\mathbf{r})]=\frac{c^{2}}{4} \nabla^{2} \hat{p}(\mathbf{r}, s) .
$$

Let us next address Laplace inversion. With the standard initial conditions, ${ }^{2}$

$$
p(\mathbf{r}, 0)=\delta(\mathbf{r}),\left.\quad \frac{\partial p(\mathbf{r}, t)}{\partial t}\right|_{t=0}=0,
$$

and the Laplace inversion formulas [47],

$$
\begin{aligned}
\mathcal{L}^{-1}\left\{s^{2} \hat{p}(\mathbf{r}, s)-s \delta(\mathbf{r})\right\} & =\frac{\partial^{2} p(\mathbf{r}, t)}{\partial t^{2}}, \\
\mathcal{L}^{-1}\{s \hat{p}(\mathbf{r}, s)-\delta(\mathbf{r})\} & =\frac{\partial p(\mathbf{r}, t)}{\partial t},
\end{aligned}
$$

\footnotetext{
${ }^{2}$ In a more standard derivation of the TE similar to that of Goldstein based on the persistent random walk on the line [6], the initial condition for the time derivative of $p(\mathbf{r}, t)$ comes in a natural way from the formalism (based on splitting the probability density $p$ in the sum of two densities, corresponding to the motion to the left or to the right) and when the walker can initially move to the left or right with equal probability (which in one dimension corresponds to the isotropic case). It then turns to be that the time derivative of $p$ at $t=0$ is zero (see details in Ref. [51]). Since we are dealing with a two-dimensional and isotropic walk the initial condition should be the same as for the isotropic one-dimensional walk.
} 
we see that $p(\mathbf{r}, t)$ satisfies the two-dimensional TE,

$$
\frac{\partial^{2} p}{\partial t^{2}}+\frac{1}{\tau} \frac{\partial p}{\partial t}=v^{2} \nabla^{2} p
$$

with

$$
\tau=2 / \lambda \quad \text { and } \quad v=c / 2,
$$

as the characteristic time and the characteristic velocity, respectively.

As in one and three dimensions, the TE enjoys both wave and diffusion characteristics. This duality becomes even more apparent as time progresses. Thus, as $t \rightarrow 0$, Eq. (37) reduces to the wave equation whereas as $t \rightarrow \infty$ it goes to the diffusion equation. Indeed, scaling time with $\tau$, one can easily see that $[8,10]$

$$
\frac{\partial^{2} p}{\partial t^{2}} \simeq v^{2} \nabla^{2} p \quad(t \rightarrow 0), \quad \frac{\partial p}{\partial t} \simeq D \nabla^{2} p \quad(t \rightarrow \infty),
$$

$\left(D=v^{2} \tau\right)$ which leads to

$$
\left\langle|\mathbf{r}(t)|^{2}\right\rangle \sim t^{2} \quad(t \rightarrow 0), \quad\left\langle|\mathbf{r}(t)|^{2}\right\rangle \sim t \quad(t \rightarrow \infty),
$$

showing the transition from ballistic motion to diffusive motion as time increases.

\section{Characteristic function}

The solution to the two-dimensional TE (37) with the initial conditions given by (36) has been, to our knowledge, scarcely addressed, at least, in the physics literature [52-54], moreover, the solutions presented in these references do not obey the initial conditions given in Eq. (36). ${ }^{3}$ We will not obtain here the exact expression for the PDF $p(\mathbf{r}, t)$, which is quite a difficult task. However, getting the exact characteristic function $\tilde{p}(\omega, t)$ is rather straightforward. Indeed, in FourierLaplace space, the exact solution of the TE (37) with initial conditions (36) is given by Eq. (35), which we write in the form (recall that $\lambda=\tau / 2$ )

$$
\hat{\tilde{p}}(\omega, s)=\frac{s+1 / 2 \tau+1 / 2 \tau}{(s+1 / 2 \tau)^{2}-\rho^{2}(\omega) / 4 \tau^{2}},
$$

where

$$
\rho(\omega)=\sqrt{1-(c \tau|\omega|)^{2}} .
$$
[47],

Bearing in mind property (28) and the inversion formulas

$$
\mathcal{L}^{-1}\left\{\frac{s}{s-a^{2}}\right\}=\cosh a t, \quad \mathcal{L}^{-1}\left\{\frac{1}{s-a^{2}}\right\}=\sinh a t,
$$

\footnotetext{
${ }^{3}$ We asserted, in Ref. [54], that the solution of the two-dimensional TE was not positive definite, a fact that somehow invalidated the $\mathrm{TE}$ as a proper equation for the PDF in two dimensions. However, this conclusion was hasty because the two-dimensional solution presented in Ref. [54] was ill defined for $r=c t / 2$ and did not obey the initial conditions (36). As a consequence, the question of not being positive definite is very doubtful (we will discuss this issue in a future work).
}

it is a simple matter to obtain from Eq. (39) the characteristic function,

$$
\tilde{p}(\boldsymbol{\omega}, t)=e^{-t / 2 \tau}\left[\cosh \frac{\rho(\boldsymbol{\omega}) t}{2 \tau}+\frac{1}{\rho(\boldsymbol{\omega})} \sinh \frac{\rho(\boldsymbol{\omega}) t}{2 \tau}\right] .
$$

Looking at Eqs. (40) and (41), we see that $\rho$ and, consequently, $\tilde{p}$ depend only on the modulus $\omega=|\omega|$. In this case, the PDF $p(r, t)$ is a function of the distance $r=|\mathbf{r}|$ to the origin (in accordance to the isotropic character of the model). Indeed, the two-dimensional Fourier inversion is given by

$$
\begin{aligned}
p(\mathbf{r}, t) & =\frac{1}{(2 \pi)^{2}} \int_{\mathbb{R}^{2}} e^{-i \omega \cdot \mathbf{r}} \tilde{p}(\omega, t) d^{2} \omega \\
& =\frac{1}{2 \pi} \int_{0}^{\infty} d \omega \omega \tilde{p}(\omega, t) \int_{0}^{2 \pi} e^{-i \omega r \cos \varphi} d \varphi \\
& =\frac{1}{\pi} \int_{0}^{\infty} d \omega \omega \tilde{p}(\omega, t) \int_{0}^{\pi} \cos (\omega r \cos \varphi) d \varphi
\end{aligned}
$$

which shows that $p=p(r, t)$ is a function of the distance to the origin. Taking into account the integral representation of the Bessel function of zeroth order [cf. Eq. (25)], we have

$$
p(r, t)=\int_{0}^{\infty} \omega J_{0}(\omega r) \tilde{p}(\omega, t) d \omega .
$$

Let us finally note that substituting Eq. (41) into Eq. (42) results in the following expression for the PDF:

$$
\begin{aligned}
p(r, t)= & e^{-t / 2 \tau} \int_{0}^{\infty} \omega\left[\cosh \frac{\rho(\boldsymbol{\omega}) t}{2 \tau}\right. \\
& \left.+\frac{1}{\rho(\boldsymbol{\omega})} \sinh \frac{\rho(\boldsymbol{\omega}) t}{2 \tau}\right] J_{0}(\omega r) d \omega,
\end{aligned}
$$

which, although difficult to integrate analytically, is rather straightforward for numerical work.

\section{THE FRACTIONAL ISOTROPIC WALK AND THE FRACTIONAL TELEGRAPHER'S EQUATION IN TWO DIMENSIONS}

We will now derive a two-dimensional fractional telegrapher's equation from a microscopic picture based on twodimensional random walks. The procedure is parallel to the one we used in deriving the three-dimensional TE, and its fractional generalization [10] and whose first step consists in obtaining a fractional generalization of the uniform and isotropic random walk outlined in Secs. III and IV.

\section{A. The fractional isotropic walk}

Let us first note that the fluid limit approximation for $\hat{\tilde{p}}(\boldsymbol{\omega}, s)$ given in Eq. (35) is also achieved by substituting into the general expression (19) the following expansions in the fluid limit as $s \rightarrow 0$ and $|\omega| \rightarrow 0:^{4}$

$$
\hat{\tilde{h}}(\omega, s)=1-\frac{s}{\lambda}-2\left(\frac{s}{\lambda}\right)^{2}-\frac{1}{2 \lambda^{2}}|\omega|^{2} c^{2} \cdots,
$$

\footnotetext{
${ }^{4}$ In order to derive Eq. (44), we start from Eq. (33) and follow the same procedure as detailed in Ref. [10] and similarly for the derivation of Eq. (45).
} 
and

$$
\hat{\tilde{H}}(\omega, s)=\frac{1}{\lambda}\left(1+\frac{2 s}{\lambda}\right) \cdots
$$

Indeed, from Eq. (44), we write

$$
1-\hat{\tilde{h}}(\boldsymbol{\omega}, s)=\frac{s}{\lambda}+2\left(\frac{s}{\lambda}\right)^{2}+\frac{1}{2 \lambda^{2}}|\omega|^{2} c^{2} \cdots .
$$

Dividing the expression for $\hat{H}(\omega, s)$ given in Eq. (45) by Eq. (46) as required by Montroll-Weiss equation (19) and rearranging terms results in the fluid limit approximation given in Eq. (35) as the reader can easily check.

We are now ready to get a fractional generalization of the two-dimensional isotropic random walk. Thus, and looking at Eq. (44), we first propose the following expansion for the sojourn density in the fluid limit:

$$
\hat{\tilde{h}}(\omega, s)=1-(T s)^{\alpha}-2(T s)^{2 \alpha}-\frac{1}{2}(L|\omega|)^{2 \gamma} \ldots
$$

$(s,|\omega| \rightarrow 0)$, where $0<\alpha \leqslant 1,0<\gamma \leqslant 1$ and $T>0$ and $L>0$ are arbitrary parameters. $T$ defines a characteristic time, and $L$ is a characteristic length.

In addition to Eq. (47), we also need to assume a fractional approximation for the function $\hat{\tilde{H}}(\omega, s)$. To this end, we average Eqs. (6) and (7) over all directions $\varphi$ with the result (in Laplace space),

$$
\int_{\mathbb{R}^{2}} \hat{h}(\mathbf{r}, s) d^{2} \mathbf{r}=\hat{\psi}(s), \quad \int_{\mathbb{R}^{2}} \hat{H}(\mathbf{r}, s) d^{2} \mathbf{r}=\hat{\Psi}(s),
$$

or in terms of Fourier transforms,

$$
\hat{\tilde{h}}(\omega=0, s)=\hat{\psi}(s), \quad \hat{H}(\omega=0, s)=\hat{\Psi}(s) .
$$

But $\hat{\Psi}(s)=[1-\hat{\psi}(s)] / s$ [cf. Eq. (5) ], and, hence,

$$
\hat{\tilde{H}}(\omega=0, s)=\frac{1}{s}[1-\hat{\tilde{h}}(\omega=0, s)] .
$$

Introducing Eq. (47) into this expression yields

$$
\hat{H}(\omega=0, s)=T^{\alpha} s^{\alpha-1}+2 T^{2 \alpha} s^{2 \alpha-1},
$$

which, as in the three-dimensional case, leads us to conjecture the following fluid limit approximation:

$$
\hat{H}(\omega, s) \simeq T^{\alpha} s^{\alpha-1}+2 T^{2 \alpha} s^{2 \alpha-1} \cdots
$$

$(s \rightarrow 0,|\omega| \rightarrow 0)$. Note that this is only a conjecture since the approximation (48) might have depended on $\omega$ as well [9].

Substituting Eqs. (47) and (48) into the Montroll-Weiss equation (19) and reorganizing terms yields

$$
\hat{\tilde{p}}(\boldsymbol{\omega}, s)=\frac{s^{\alpha-1}\left(s^{\alpha}+1 / \tau\right)}{s^{2 \alpha}+s^{\alpha} / \tau+v^{2}|\omega|^{2 \gamma}},
$$

where

$$
\tau \equiv 2 T^{\alpha}, \quad v \equiv \frac{1}{2}\left(L^{\gamma} / T^{\alpha}\right)
$$

$(0<\alpha \leqslant 1,0<\gamma \leqslant 1)$. The parameters $\tau$ and $v$ can be considered as a fractional characteristic time and a fractional characteristic velocity, respectively. This equation describes a fractional isotropic random walk in two dimensions and allows us to derive the corresponding FTE.

\section{B. The fractional telegrapher's equation}

Before deriving a fractional generalization of the TE in two dimensions, we briefly recall the basic definitions of the necessary fractional derivatives.

The Caputo fractional derivative of order $\beta>0$ of a function $\phi(t)$ is defined by the functional [21,55-58],

$$
\frac{\partial^{\beta} \phi(t)}{\partial t^{\beta}}= \begin{cases}\frac{1}{\Gamma(n-\beta)} \int_{0}^{t} \frac{\phi^{(n)}\left(t^{\prime}\right) d t^{\prime}}{\left(t-t^{\prime}\right)^{1+\beta-n}}, & n-1<\beta<n, \\ \phi^{(n)}(t), & \beta=n\end{cases}
$$

$(n=1-3, \ldots)$, where $\phi^{(n)}(t)$ denotes the $n$th derivative with respect to $t$. From this definition, the Laplace transform of the Caputo derivative is found to be $[8,56]$

$$
\mathcal{L}\left\{\frac{\partial^{\beta} \phi(t)}{\partial t^{\beta}}\right\}=s^{\beta} \hat{\phi}(s)-s^{\beta-1} \phi(0)-\sum_{j=1}^{n-1} s^{\beta-1-j} \phi^{(j)}(0)
$$

$(n=1-3, \ldots, n-1<\beta<n)$. Here, $\mathcal{L}\{\cdot\}$ stands for the Laplace transform and $\hat{\phi}(s)=\mathcal{L}\{\phi(t)\}$.

The second type of fractional derivative that is needed is the Riesz-Feller Laplacian of order $\beta(0<\beta \leqslant 2)$ of a function $g(\mathbf{r})$ such that $g(\mathbf{r}) \rightarrow 0$ as $|\mathbf{r}| \rightarrow \infty$. There are several equivalent ways to define it [56], although one of the simplest and most operative definitions is obtained through Fourier analysis. We, thus, define [21]

$$
\nabla^{\beta} g(\mathbf{r})=\mathcal{F}^{-1}\left\{-|\omega|^{\beta} \tilde{g}(\omega)\right\}
$$

$(0<\beta \leqslant 2)$, where $\mathcal{F}^{-1}\{\cdot\}$ stands for the inverse Fourier transform, and

$$
\tilde{g}(\boldsymbol{\omega})=\int_{\mathbb{R}^{2}} e^{i \omega \cdot \mathbf{r}} g(\mathbf{r}) d^{2} \mathbf{r}
$$

is the direct transform.

From Eq. (49), the FTE is readily obtained as follows. We rewrite Eq. (49) as:

$$
\left(s^{2 \alpha}+\frac{1}{\tau} s^{\alpha}+v^{2}|\omega|^{2 \gamma}\right) \hat{\tilde{p}}(\boldsymbol{\omega}, s)=s^{2 \alpha-1}+\frac{1}{\tau} s^{\alpha-1} .
$$

Fourier inverting and recalling that $\mathcal{F}^{-1}\{1\}=\delta(\mathbf{r})$, we have

$$
\left(s^{2 \alpha}+\frac{1}{\tau} s^{\alpha}-v^{2} \nabla^{2 \gamma}\right) \hat{p}(\mathbf{r}, s)=\left(s^{2 \alpha-1}+\frac{1}{\tau} s^{\alpha-1}\right) \delta(\mathbf{r}),
$$

where $\nabla^{2 \gamma}$ is the two-dimensional fractional Laplacian of order $2 \gamma$.

The Laplace inversion of this equation finally results in (see Refs. [10] and [9] for details)

$$
\frac{\partial^{2 \alpha} p}{\partial t^{2 \alpha}}+\frac{1}{\tau} \frac{\partial^{\alpha} p}{\partial t^{\alpha}}=v^{2} \nabla^{2 \gamma} p
$$

which is the FTE in two dimensions with $\tau$ as a fractional timescale and $v$ as a fractional velocity [cf. Eq. (50)].

Such as the ordinary TE (37), the FTE (54) also possesses both wave and diffusion characteristics. Thus, the joint limits $\tau \rightarrow 0$ and $v \rightarrow \infty$ with $\tau v^{2} \rightarrow D$ finite turns Eq. (54) into a two-dimensional fractional diffusion equation,

$$
\frac{\partial^{\alpha} p}{\partial t^{\alpha}}=D \nabla^{2 \gamma} p
$$


whereas the limit $\tau \rightarrow \infty$ with $v$ finite reduces Eq. (54) into the fractional diffusion-wave equation,

$$
\frac{\partial^{2 \alpha} p}{\partial t^{2 \alpha}}=v^{2} \nabla^{2 \gamma} p
$$

Let us finally mention that the FTE (54) leads to the fractional diffusion equation (55) as $t \rightarrow \infty$ and to the fractional wave equation (56) as $t \rightarrow 0$, regardless of the values of the fractional time $\tau$ and the fractional velocity $v$ [9].

\section{CONCLUDING REMARKS}

Following the approach established in Ref. [10] for the three-dimensional case, we have here obtained the twodimensional telegrapher's equation (ordinary and fractional) starting with an isotropic and uniform random walk on the plane. The model, which is a two-dimensional generalization of the persistent random walk on the line, is based on multistate random walks with a continuous number of states representing the different directions that the walker can take. When, at every point, the possible directions are independent and do not depend on the orientation and position (isotropy and homogeneity), the general equations for the probability density function of the random walker can be exactly solved in Fourier-Laplace space.

We remark once more that the two-dimensional case cannot be recovered from the three-dimensional walk as a particular case and, although the two- and three-dimensional models have many common characteristics, the quantitative results obtained differ.

Either in two or three dimensions the isotropic and uniform model is suitable for modeling the transport of particles experiencing elastic collisions with fixed centers randomly distributed. Two-dimensional models are particularly appropriate in the study of transport properties on surfaces and near interfaces [30-35].

From the random walk model, we have been able to derive a two-dimensional telegrapher's equation in the so-called fluid limit approximation, which involves long times and long distances and represents a mesoscopic approximation, halfway between the microscopic description of the isotropic random walk and the macroscopic description of any phenomenological setting. With this result, we have shown that the telegrapher's equation - in one, two, and three dimensions - can be derived from a random walk model. In one dimension, this possibility was proved many years ago by Goldstein [6], who showed that the persistent random walk on the line leads to the one-dimensional telegrapher's equation [6]. As pointed out in Sec. I, many attempts to extend Goldstein's result to higher dimensions in a natural way had failed essentially because of the great difficulty of generalizing persistence in dimensions greater than one [17,23-29].

In any number of dimensions (one, two, or three), the telegrapher's equation obtained has the standard form

$$
\frac{\partial^{2} p}{\partial t^{2}}+\frac{1}{\tau} \frac{\partial p}{\partial t}=v^{2} \nabla^{2} p .
$$

However, the relationship between the characteristic velocity $v$ with the speed $c$ of the particle between consecutive scatterings differs depending on the dimensionality. We, thus, have $v=c$ in one dimension [7], whereas

$$
v=c / 2 \quad \text { and } \quad v=c / \sqrt{6}
$$

in two and three dimensions, respectively (see Eq. (38) and Ref. [10]). In all three cases, the relationship between the characteristic time $\tau$ and the average time that the walker moves along the same direction $\lambda^{-1}$ is given by $\tau=2 \lambda^{-1}$.

As we did in one and three dimensions (Refs. $[9,10])$, we have also generalized the isotropic walk and, subsequently, the telegrapher's equation to account for anomalous transport in two dimensions. Again, the fractional telegrapher's equation formally has the same form regardless the number of dimensions,

$$
\frac{\partial^{2 \alpha} p}{\partial t^{2 \alpha}}+\frac{1}{\tau} \frac{\partial^{\alpha} p}{\partial t^{\alpha}}=v^{2} \nabla^{2 \gamma} p
$$

$(0<\alpha \leqslant 1,0<\gamma \leqslant 1)$, although, as before, the relationship between the fractional velocity $v$ and the characteristic time $T$ and length $L$ of the fractional random walk depends on the dimensionality. We, thus, have $v=L^{\gamma} / T^{\alpha}$ in one dimension [9], and

$$
v=\frac{1}{2}\left(L^{\gamma} / T^{\alpha}\right), \quad v=\frac{1}{\sqrt{6}}\left(L^{\gamma} / T^{\alpha}\right),
$$

in two and three dimensions, respectively (cf. Eq. (50) and Ref. [10]).

Let us finally mention that, as in the one-dimensional case [9] or for the ordinary telegrapher's equation [cf. Eq. (41)], an exact solution for the characteristic function $p(\omega, t)$ of the two-dimensional fractional telegrapher's equation can be obtained along with some asymptotic and approximate expressions. However, for the sake of simplicity and brevity, we have not treated them here, and we refer the interested reader to the work of Mainardi and collaborators [55,57-59] on solutions of fractional diffusion and fractional wave-diffusion equations as well as to Orsingher and collaborators [60-62] on several kinds of solutions of the fractional telegrapher's equation.

\section{ACKNOWLEDGMENTS}

J.M. acknowledges partial financial support from MINECO under Contract No. FIS2016-78904-C3-2-P and from AGAUR under Contract No. 2017SGR1064.
[1] G. H. Weiss, Physica A (Amsterdam) 311, 381 (2002).

[2] J. Masoliver and G. H. Weiss, Eur. J. Phys. 17, 190 (1996).
[3] D. D. Joseph and L. Preziosi, Rev. Mod. Phys. 61, 41 (1989); 62, 375 (1990). 
[4] D. Jou, J. Casas-Vázquez, and G. Lebon, Extended Irreversible Thermodynamics, 4th ed. (Springer, Berlin, 2010).

[5] V. Méndez, D. Campos, and W. Horsthemke, Phys. Rev. E 90, 042114 (2014).

[6] S. Goldstein, Q. J. Mech. Appl. Math. 4, 129 (1951).

[7] J. Masoliver, K. Lindenberg, and G. H. Weiss, Physica A (Amsterdam) 157, 891 (1989).

[8] J. Masoliver and K. Lindenberg, Eur. Phys. J. B 90, 107 (2017).

[9] J. Masoliver, Phys. Rev. E 93, 052107 (2016).

[10] J. Masoliver, Phys. Rev. E 96, 022101 (2017).

[11] M. A. Olivares-Robles and L. S. Garcia-Colin, Phys. Rev. E 50, 2451 (1994).

[12] L. S. Garcia-Colin and M. A. Olivares-Robles, Physica A (Amsterdam) 220, 165 (1995).

[13] M. A. Olivares-Robles and L. S. Garcia-Colin, J. Non-Equilib. Thermodyn. 21, 361 (1996).

[14] J. J. Duderstadt and W. R. Martin, Transport Theory (Wiley, New York, 1979).

[15] A. J. Ishimaru, Appl. Opt. 28, 2210 (1989).

[16] S. I. Heizler, Nucl. Sci. Eng. 166, 17 (2010).

[17] A. V. Plyukhin, Phys. Rev. E 81, 021113 (2010).

[18] D. J. Durian and J. Rudnick, J. Opt. Soc. Am. A 14, 235 (1997).

[19] P. A. Lemieux, M. U. Vera, and D. J. Durian, Phys. Rev. E 57, 4498 (1998).

[20] D. J. Durian and J. Rudnick, J. Opt. Soc. Am. A 16, 837 (1999).

[21] R. Balescu, Chaos Solitons Fractals 34, 62 (2007).

[22] G. H. Weiss, Aspects and Applications of the Random Walk (North-Holland, Amsterdam, 1994).

[23] J. Masoliver, J. M. Porrà, and G. H. Weiss, Physica A (Amsterdam) 182, 593 (1992).

[24] J. M. Porrà, J. Masoliver, and G. H. Weiss, Physica A (Amsterdam) 218, 229 (1995).

[25] S. Godoy and L. S. García-Colín, Phys. Rev. E 55, 2127 (1997).

[26] M. Boguñá, J. M. Porrà, and J. Masoliver, Phys. Rev. E 58, 6992 (1998).

[27] A. D. Kolesnik and E. Orsingher, J. Appl. Prob. 42, 1168 (2005).

[28] E. Orsingher and A. De Gregorio, J. Theor. Prob. 20, 769 (2007).

[29] A. Kolesnik and M. A. Pinsky, J. Stat. Phys. 142, 828 (2011).

[30] S. Hadsegawa and F. Grey, Surf. Sci. 500, 84 (2002).

[31] G. Antczak and G. Ehrlich, Phys. Rev. Lett. 92, 166105 (2004).

[32] J. M. Sancho, A. M. Lacasta, K. Lindenberg, I. M. Sokolov, and A. H. Romero, Phys. Rev. Lett. 92, 250601 (2004).

[33] J. Ferron, L. Gomez, J. J. de Miguel, and R. Miranda, Phys. Rev. Lett. 93, 166107 (2004).

[34] H. Bulou and J.-P. Bucher, Phys. Rev. Lett. 96, 076102 (2006).

[35] M. J. Skaug, A. M. Lacasta, L. Ramirez-Piscina, J. M. Sancho, K. Lindenberg, and D. K. Schwartz, Soft Matter 10, 753 (2014).
[36] D. C. Senft and G. Ehrlich, Phys. Rev. Lett. 74, 294 (1995).

[37] T. R. Linderoth, S. Horch, E. Laegsgaard, I. Stensgaard, and F. Besenbacher, Phys. Rev. Lett. 78, 4978 (1997).

[38] S.-M. Oh, S. J. Koh, K. Kyuno, and G. Ehrlich, Phys. Rev. Lett. 88, 236102 (2002).

[39] D. Krapf and R. Metzler, Phys. Today 72(9), 48 (2019).

[40] P. Schuck and H. Zhao, Methods Mol. Biol. 627, 15 (2010).

[41] A. Compte and R. Metzler, J. Phys. A 30, 7277 (1997).

[42] R. Metzler and A. Compte, Physica A (Amsterdam) 268, 454 (1999).

[43] R. Metzler and T. F. Nonnenmacher, Phys. Rev. E 57, 6409 (1998).

[44] E. W. Montroll and G. H. Weiss, J. Math. Phys. 6, 167 (1965).

[45] E. W. Montroll and M. F. Shlesinger, in Studies in Statistical Mechanics, edited by J. L. Lebowitz and E. W. Montroll (NorthHolland, Amsterdam, 1984), Vol. 11.

[46] W. Magnus, F. Oberhettinger, and R. P. Soni, Formulas and Theorems for the Special Functions of Mathematical Physics (Springer-Verlag, Berlin/New York, 1966).

[47] G. E. Roberts and H. Kaufman, Table of Laplace Transforms (W. B. Saunders, Philadelphia, 1966).

[48] R. Gorenflo, F. Mainardi, and A. Vivoli, Chaos, Solitons \& Fractals 34, 87 (2007).

[49] W. Feller, An Introduction to Probability Theory and its Applications (Wiley, New York, 1971), Vol. II.

[50] R. A. Handelsman and J. S. Lew, SIAM J. Math. Anal. 5, 425 (1974).

[51] J. Masoliver, J. M. Porrà, and G. H. Weiss, Phys. Rev. E 48, 939 (1993).

[52] P. M. Morse and H. Feshbach, Methods of Theoretical Physics (McGraw-Hill, New York, 1953), Vol. I.

[53] E. Orsingher, J. Appl. Prob. 23, 385 (1986).

[54] J. M. Porrà, J. Masoliver, and G. H. Weiss, Phys. Rev. E 55, 7771 (1997).

[55] R. Gorenflo and F. Mainardi, in Fractals and Fractional Calculus in Continuum Mechanics, edited by A. Carpinteri and F. Mainardi (Springer, Berlin, 1997).

[56] I. Podlubny, Fractional Differential Equations (Academic, San Diego, 1999).

[57] F. Mainardi, Appl. Math. Lett. 9, 23 (1996).

[58] F. Mainardi, Y. Luchko, and G. Pagnini, Fract. Calc. Appl. Anal. 4, 153 (2001).

[59] F. Mainardi and G. Pagnini, J. Comp. Appl. Math. 207, 245 (2007).

[60] E. Orsingher and X. Zhao, Chinese Ann. Math. B 24, 45 (2003).

[61] E. Orsingher and L. Beghin, Probab. Theory Relat. Fields 128, 141 (2004).

[62] M. D’Ovidio, E. Orsingher, and B. Toaldo, Stochastic Anal. Appl. 32, 1009 (2014). 